\title{
D-25 LAMINAR FLOW MODELLING OF INITIAL SALT DEFORMATION
}

A. EBLAOU and G. DEBONO

Malta University, Physics Department, Msida, Matta

\section{Summary:}

Although salt structures can assume complex geometries, their gross regional distribution in a salt province is often observed to follow a more simple and regular pattern that can be mathematically described in terms of an initial horizontal salt flow caused by loading variations in the overburden.

The theory of laminar flow within a uniform horizontal layer of salt subjected to two load gradients is here developed using basic hydrodynamic principles. The exertion of a linear load gradient is seen to trigger the initial generation of equally spaced salt swells which can then develop further into complex structures under the influence of buoyancy. In contrast, the exertion of a radial positive load gradient induces a series of circular swells with amplitudes decaying away from the location of minimum load.

For a given load gradient, the shape, magnitude and spacing of these salt deformations are seen to depend on the density, viscosity and thickness of the salt, and on a time factor. They are also comparable to the shape and distribution of salt structures observed in nature. Scaled physical models using dry fine quartz sand to simulate the overburden and viscose asphalt to represent the salt layer were also used to test the theoretical predictions.

These basic analytical considerations are finally used to explain the occurrence and distribution of salt structures in a basin in Northwest Germany. The linearity, trend and spacing of a set of parallel salt walls found in the basin are explained by a progradational overburden which can be modelled by the linear load gradient algorithm.

The petroliferous nature of structures influenced by the salt deformation makes these analytical considerations valuable exploration tools capable of predicting the gross distribution of saltinduced structures in other regions of the world.

\section{Introduction:}

Empirical relations have been established as early as 1960 to account for some of the simpler geometrical properties of salt domes, plugs or stocks, such as their spacing. More theoretical formulations for the growth of model diapirs were proposed in 1975. This and other similar models are based on a two layer system of liquids in which the denser medium overlies the less dense layer thus creating an initial hydraulic system which, though at rest, is in an unstable equilibrium and is upset by the slightest perturbation to induce the upward movement of the less dense material through the denser medium. The theoretical basis for the equilibrium instability condition is Rayleigh-Taylor classical hydraulic equation of instability. Unless one is dealing 
with deep seated salt, and hence relatively hot, the relevance of this theory to the flow of salt is very questionable as it applies only to viscous liquids. Considerations based on plastic flow appear to be more realistic. On the basis of such considerations it was shown that the spacing of salt plugs is not, in general controlled by the Rayleigh-Taylor viscous instability, but is related to the dimensions of the salt plug and the permissible limits of subsidence of the roof rock. These considerations, although able to describe the spacing of plugs, have many shortcomings. A major failure is that they do not approach the problem as a whole but treat each structure separately. No account for the distribution of structures can be made.

The problem is overcome by considering the salt layer under a given environment as a whole. An analogous case is encountered in solving the problem of glacial rebound in geophysics. This holistic approach is used in the forthcoming treatment of salt deformation in an attempt to define the initial deformation of salt which can then develop further into complex structures under the influence of buoyancy

\section{Theory:}

The theory of laminar flow within a uniform horizontal layer of salt subjected to two load gradients is here developed using basic hydrodynamic principles. The exertion of a linear load gradient is considered first and is then extended to a positive radial load gradient

\section{(i) Linear load gradient algorithm-}

A horizontal layer of salt of thickness $H$ overlain by a uniform 2-D wedge of sediments is subjected to a linear pressure gradient due to lateral variations in thickness or density of overburden This pressure gradient tends to cause the salt to flow laterally by virtue of its finite

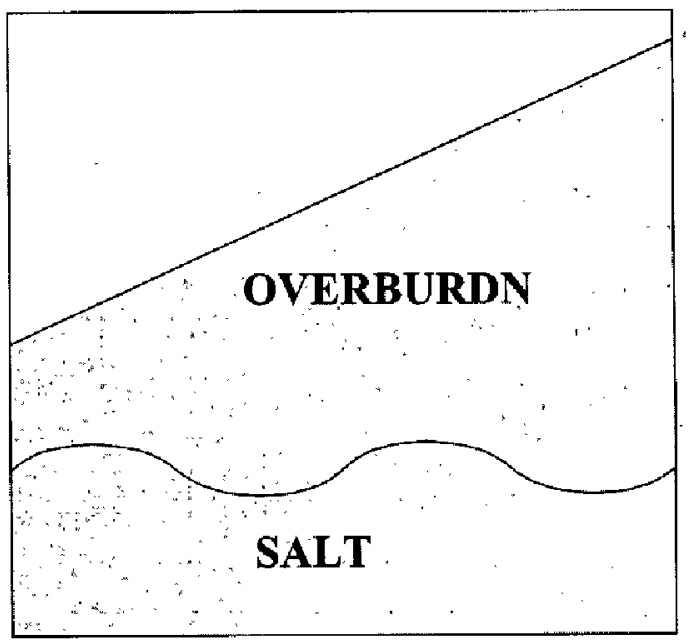

viscosity $\eta$ towards the direction of least pressure. As a result, the upper boundary of the salt layer undergoes a vertical displacement $Z$ which is defined by the differential equation:

$\frac{\mathrm{d}^{2} Z}{\mathrm{dx}^{2}}=\frac{-12 \eta}{\rho g \mathrm{H}^{3} \tau} \cdot \mathrm{Z}$

where $\rho$ is the density of the salt, $x$ is the horizontal distance along the top of the salt layer, $g$ is the gravity and $\tau$ is a time constant. 
The solution to this equation is simply

$$
Z=A \operatorname{Sin}(\omega x)
$$

where $\omega=\underline{\sqrt{ } 12 \eta}$ and $A$ is a constant and $\tau$ is a time factor.

$$
\sqrt{\rho g H^{3} \tau}
$$

The salt is assumed to behave as a perfect Newtonian viscous fluid and the lower boundary of the salt layer is assumed to behave rigidly. It is further assumed that the horizontal dimensions of the salt layer is much larger than the vertical dimensions so that the viscous drag of the horizontal flow limits the rate of vertical deformation.

\section{(ii) Radial load gradient algorithm-}

When the horizontal layer of salt is overlain by sediments in the form of a 3D hemispherical depression such that a positive radial pressure gradient is exerted on the salt layer, with minimum pressure coinciding with the centre of the depression, the upper boundary of the salt layer undergoes a vertical displacement $Z$ which is defined by the differential equation

$$
\frac{\mathrm{d}^{2} \mathrm{Z}}{\mathrm{dr}^{2}}+\frac{1}{\mathrm{r}} \frac{\mathrm{dZ}}{\mathrm{dr}}+\frac{12 \eta}{\rho g \mathrm{H}^{3} \tau} \mathrm{Z}=0
$$

where $r$ is the horizontal distance along the top of the salt layer from the centre of the depression. This Bessel equation for $\mathrm{n}=0$ i.e

$$
\frac{\mathrm{d}^{2} \mathrm{Z}}{\mathrm{dx}^{2}}+\frac{1}{\mathrm{x}} \frac{\mathrm{dZ}}{\mathrm{dx}}+1-\underline{\mathrm{n}}^{2} \quad \mathrm{Z}=0
$$

where $\mathrm{x}=\underline{\sqrt{ } 12 \eta} \mathrm{r}$ and $\tau$ is a time factor.

$$
\sqrt{\rho g H^{3} \tau}
$$

A realizable solution to this equation is the zero order Bessel function of the first kind, i.e.

$$
\mathbf{Z}=\mathbf{Z}_{\mathbf{r}=0} \mathbf{J}_{\mathbf{0}}\{\mathbf{r}\}
$$

The zeros for $J_{o}\{r\}$ occur at $r=2.405,5,520,8.654$.. etc i.e. whenever $r=(n-1 / 4) \pi, n \in N$

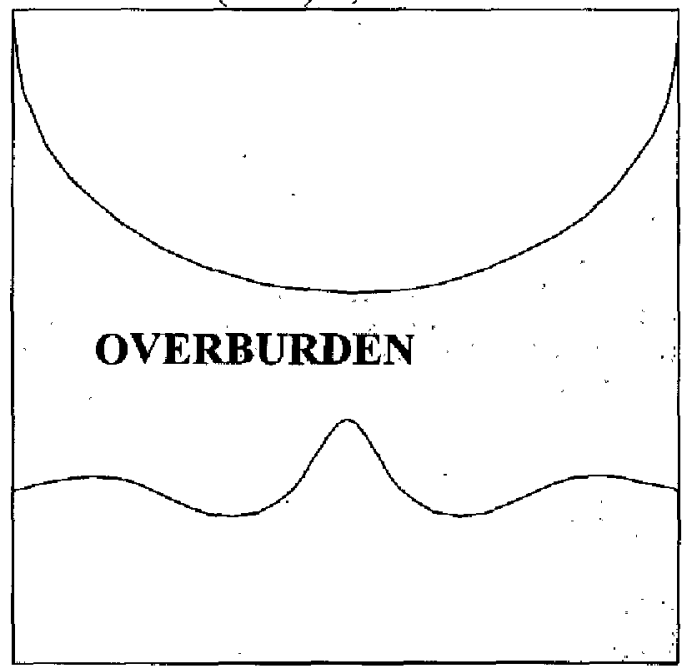




\section{Physical models and geological analogues:}

Scaled physical models were built to investigate the initial deformations of a plastic horizontal layer subjected to the two differential loading regimes, one representing a prograding geological template and the other a hemispherical basin. Dry, fine quarts sand was used to simulate the overburden while viscose asphalt at room temperature simulated the salt. All models were carried out in glass or perspex walled containers to enable the visual observation of deformations. The deformations of the top layer of asphalt were observed to match the predictions of the analytical relations described above to a remarkable degree.

These basic analytical considerations are used to explain the occurrence and distribution of salt walls in a well known salt basin in Northwest Germany. These salt walls average $4 \mathrm{~km}$ in width, may reach a length of over $120 \mathrm{~km}$ and are arranged parallel to each other and 8 to $10 \mathrm{~km}$ apart. A prograding sedimentation overlying the salt is believed to have generated these walls by causing the salt to flow laterally and deform. In particular, the linearity, trend and spacing of the salt walls can be explained by simulating the initial salt deformation by the linear load gradient algorithm.

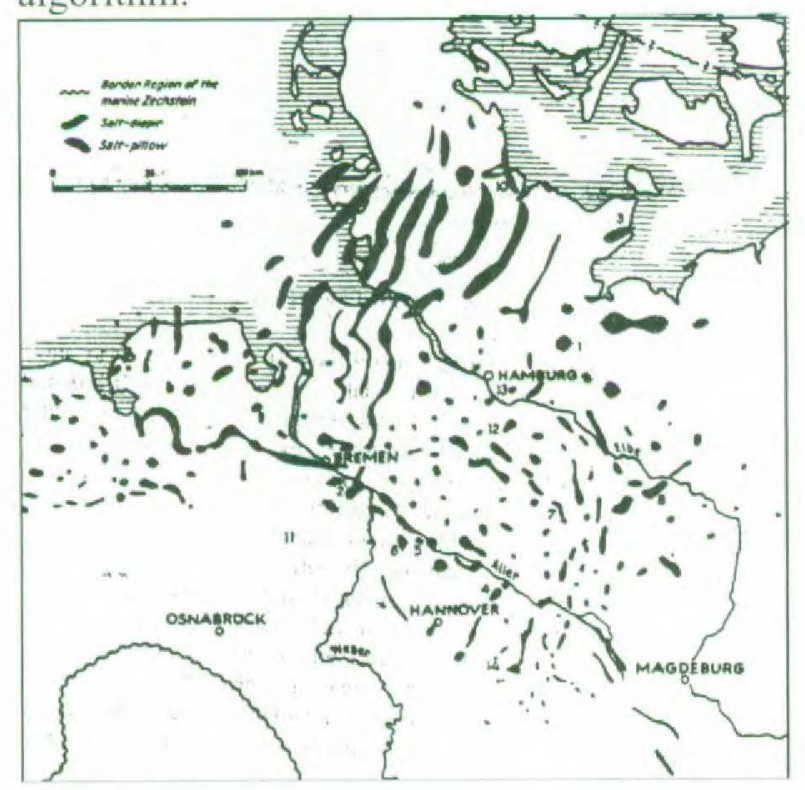

\section{Conclusions:}

Analytical relations have been established to define the initial deformation of the top boundary of a horizontal salt layer subjected to load gradients attributed to overburdens of simple geometric forms. Scaled physical models using dry fine quartz sand to simulate the overburden and asphalt to represent the salt layer agree with the theoretical predictions.

It is seen that the exertion of a linear load gradient on a horizontal uniform layer of salt gives rise to deformations in the upper layer in the form of a series of regular parallel swells and pillows with spacing depending on the density, viscosity and thickness of the salt, and a time factor. It is also seen that the exertion of a positive radial load gradient gives rise to deformations in the upper layer of the salt in the form of a series of circular swells with amplitudes decaying away from the centre and spacing depending on the density, viscosity and thickness of the salt, and a time factor.

These basic analytical considerations can successfully explain the occurrence and distribution of salt walls in a well known salt basin in Northwest Germany and can be applied to study the gross distribution of salt-induced structures in other regions of the world. 